\title{
EXTENDING CTF MODELING CAPABILITIES TO SFRs AND VALIDATION AGAINST SHRT TESTS
}

\author{
Ahmed Aly, Agustin Abarca, Maria Avramova, Kostadin Ivanov \\ North Carolina State University \\ 3140 Burlington Engineering Labs \\ 2500 Stinson Drive \\ Raleigh, NC, USA 27695-7909 \\ amaly@ncsu.edu, aabarca@ncsu.edu, mnavramo@ncsu.edu, knivanov@ncsu.edu
}

\begin{abstract}
The utilization of liquid metals as coolants for fast reactors brings several economical and practical advantages that lead to a sustainable future for nuclear energy. Molten sodium is used as a coolant in Sodium Fast Reactors (SFRs). Sodium is relatively cheaper than other metal coolants. It requires lower pumping power, causes less neutron moderation and it is noncorrosive to the fuel cladding. The SFR hexagonal subassemblies are relatively smaller than Light Water Reactors (LWRs) subassemblies. The differences in the geometrical design of SFRs compared to LWRs lead to different physical behavior of the coolant. Several models and correlations particular to sodium were implemented in thermal-hydraulics (TH) computer codes in order to model the coolant behavior accurately. CTF is a subchannel TH code that was designed and validated for LWRs. In this work, the capabilities of the code were extended to SFRs by incorporating sodium coolant properties and correlations for friction factor, flow mixing coefficient and conduction heat transfer. The code was then validated against selected steady state data from the Experimental Breeder Reactor II Shutdown Heat Removal Tests SHRT-17 and SHRT-45R. CTF was used to simulate the instrumented subassemblies XX09 and XX10. The results demonstrate the capability of CTF to model SFRs. Code validation is currently being extended to the transient phases of the SHRT experiments.
\end{abstract}

KEYWORDS: CTF, SFR, SHRT, EBR-II

\section{INTRODUCTION}

Liquid metal coolants are used in fast reactor cores to transfer the heat generated in the fuel elements to secondary coolant loops and eventually to generate steam that is used to drive turbines to produce electrical power as in any conventional nuclear power plant [1]. The utilization of liquid metals as coolants for Generation IV fast reactors is a promising feature. It assures a place in the future for nuclear power plants as a sustainable, economical and a clean environmentally friendly source of energy. It can sustain the ascending needs of electricity in the rapidly growing industrial world and the domestic necessities of the modern life.

The high thermal conductivity and the high melting point of metal coolants allow the operation of Liquid Metal Fast Breeder Reactors (LMFBRs) at relatively higher power densities in a smaller reactor volume. 
They also allow the reactor to operate at near-atmospheric pressure ranges which makes the design process less complicated [2]. Sodium distinguish itself as an excellent type of coolant for fast reactors with its melting point of $97^{\circ} \mathrm{C}$. It is relatively cheaper than other metal coolants, requires lower pumping power, has lower moderation power and it is non-corrosive to the stainless-steel cladding [1].

The higher power densities in a smaller volume hexagonal fuel assembly and the mechanical support of the fuel pins by the wire wrapping differentiates SFRs assemblies from the rectangular, gird spacer supported LWR assemblies [3]. The coolant properties, such as the dynamic viscosity and specific heat, are different. The non-dimensional Reynolds number, which differentiates the laminar and turbulent flow as well as the transition between them, are different for water and sodium [4]. This affects the pressure drop quantification along the fuel rod length. The drag forces across the wire wrap lead to enhanced flow mixing along the whole length of the fuel [5]. The heat conduction in the sodium is much higher than that of the water and should be taken into consideration especially in the case of natural circulation [6]. Thermal-hydraulic codes must consider these factors when modeling SFR subassemblies.

CTF [7] is a version of COBRA-TF (COolant Boiling in Rod Array - Two Fluid) subchannel code that is being jointly developed and maintained by the Reactor Dynamics and Fuel Modeling Group at North Carolina State University and the Oak Ridge National Laboratory within the U.S. Department of Energy Consortium for Advanced Simulations of Light Water Reactors (CASL) project. The code is extensively validated for LWRs. The code capabilities were recently extended to modelling SFRs. The coolant properties were compiled and encoded in CTF. Cheng and Todreas correlations for the flow mixing coefficient and the friction factors [8] were added to the code. The heat conduction across the gaps between subchannels, which is neglected for water, is now introduced to the energy conservation equation when sodium coolant is modeled.

CTF was validated against the Shutdown Heat Removal Tests SHRT-17 and SHRT-45. The tests were safety experiments performed in the Experimental Breeder Reactor II (EBR-II) [9] to demonstrate the effects of natural circulations and negative reactivity feedbacks in keeping the reactor safe. Two subassemblies XX09 and XX10 were instrumented with thermocouples at various axial and radial locations in the core to provide data for code validation in the benchmark [10].

\section{IMPLEMENTAION OF SODIUM COOLANT MODELING IN CTF}

Several source subroutines in CTF were modified to incorporate the SFR modeling aspects to the code. The modifications included the coding of the coolant properties and correlations in a sodium-specific module that includes correlations or function that incorporates the following functions or properties.

\subsection{Sodium Coolant Properties}

The sodium properties correlations were provided by the Gesellschaft für Anlagen - und Reaktorsicherheit (GRS) [11]. They were assembled from various academic and research institutions. The liquid sodium properties are based on correlations derived specifically for liquid sodium [12]. CTF was designed originally for water coolant and performs computations at saturated and vapor phases. There were limited sets of correlations for saturated or vapor sodium. This means that the code will crash when performing computations at saturated or vapor phases. To overcome this issue, when a correlation is not available for sodium coolant, the code calls the liquid sodium correlation and compute the properties at saturation temperatures. The Nusselt number was computed based on the correlation by Borishanskii et al. [6]. 


\subsection{Coolant Mixing Coefficients}

Cheng \& Todreas correlation [8] was used to model the flow mixing coefficients between subchannels. The correlation differentiates between the mixing at interior channels and edge channels. For interior channels where the mixing is due to effective eddy diffusivity,

$$
\epsilon^{*}=C_{m}\left(\frac{A_{r 1}}{A_{1}^{\prime}}\right)^{1 / 2} \tan (\theta) .
$$

For mixing at the edge channels where the mixing is governed by the transverse flow velocities,

$$
\mathrm{C}_{1 \mathrm{~L}}=C_{S}\left(\frac{A_{r 2}}{A_{2}^{\prime}}\right)^{1 / 2} \tan (\theta) \text {. }
$$

Where $C_{m}$ and $C_{s}$ are empirically determined constants, $A_{r 1,2}$ are wire projected areas for interior and edge channels respectively, $A_{1,2}^{\prime}$ are bare rods channel flow areas for interior an edge subchannels respectively, $\theta$ is the angle the wire makes with vertical axis.

\subsection{Friction Factors}

The Cheng \& Todreas simplified correlation [4] is implemented in CTF to determine the friction factor. The correlation differentiates between the laminar, turbulent and the transition region in between. For the laminar region where $R e<R e_{L}$,

$$
f=\frac{C_{f l}}{R e}
$$

and for the turbulent region where $R e>R e_{T}$

$$
f=\frac{C_{f T}}{R e^{0.18}}
$$

where $R e, R e_{L}, R e_{T}$ are Reynolds number, Reynolds number upper limit for Laminar region and Reynolds number lower limit for Turbulent region respectively. $C_{f l}, C_{f T}$ are empirically determined constants for the laminar and turbulent regions respectively and are dependent on the wire lead length and geometrical parameters of the subassembly. For the transition region, an interpolation function between the laminar and the turbulent region is used to determine the friction factor.

\subsection{Heat Conduction in Sodium}

In subchannel codes, the coolant parameters are averaged over each subchannel. The heat conduction in the coolant happens across the gap between two subchannels. This is complicated to compute since the local temperatures in each subchannel separated by the gap is averaged out and the distance across the gap at which heat transfer by conduction occurs is not defined in the computational mesh [6]. Instead, the conduction heat transfer between subchannels is computed using the average temperatures in the subchannels across the centroid to centroid distance between subchannels multiplied by conduction shape factor to account for this approximation [13]. 
The conduction shape factor is geometry dependent. It is approximated in CTF using the correlation by Jeong et al. [13] to be

$$
\eta=0.777\left(\frac{P}{D}\right)\left(\frac{S}{D}\right)^{-0.263}
$$

where $\mathrm{P}$ is the fuel pitch, $\mathrm{D}$ is the fuel diameter, and $\mathrm{S}$ is the gap width.

\section{THE EBR-II SHRT BENCHMARK TESTS}

The SHRT program was initiated to provide data for computer code validation and licensing for liquid metal reactors [10]. It was performed in EBR-II operated by Argonne National Laboratory between 1964 and 1994. The nominal thermal power of the reactor was $62.5 \mathrm{MW}$ with primary sodium mass flow rate of $485 \mathrm{~kg} / \mathrm{s}$. The reactor was instrumented to measure operation parameters i.e. pressure, temperature, and mass flow rates. This allowed the data collection for validation and analysis [9].

\subsection{SHRT-17 and SHRT-45 Tests Description}

SHRT-17 was a loss of flow test. In this test, the reactor was operated at steady state power level of 57.3 MW until equilibrium condition were reached. The primary sodium flow rate was $0.54 \mathrm{~kg} / \mathrm{s}$ calibrated at $426^{\circ} \mathrm{C}[10]$. The transient was initialed by tripping the primary and intermediate sodium pumps. No auxiliary pumps were turned on which imitated the situation of a station blackout. The sodium flow coast down meant that the reactor was cooled by natural circulation. No actions were taken by the operators until the experiment was over and the reactor conditions remained within safe limits. This demonstrated the effectiveness of natural circulation characteristics in keeping the reactor safe.

SHRT-45 was a loss of flow test without protection. The control rods drivers were deactivated to prohibit control rods insertion or withdrawal by drive motors. The reactor was operated at steady state power of 60.0 MW and a primary sodium flow rate of $0.57 \mathrm{~kg} / \mathrm{s}$. Similar to the SHRT-17, the transient was initiated by tripping the primary and intermediate pumps. This time the control rods did not intervene. As the transient progressed, the reactor power decreased due to negative reactivity feedbacks and the reactor conditions were within safe limits during the whole run time of the transient. The test demonstrated the effectiveness of passive reactivity feedbacks in keeping the reactor safe during unprotected loss of flow incidents [14].

\subsection{Instrumented Subassemblies}

The subassemblies labeled XX09 and XX10 were equipped with mass flow meters and thermocouples (TC) at various radial and axial location in the core for cladding temperature measurements and the coolant inlet temperatures. They were inserted in locations reserved originally for control rods. Subassembly XX09 is similar to the regular fuel driver assemblies but contained sixty-one rods instead of ninety-one. Two of those sixty-one rods locations were reserved for flow meter conduits. Subassembly XX10 contained eighteen stainless steel pins and one tube reserved for flow meter instruments. The two subassemblies were inserted into a guide thimble of the same size as the driver assemblies. Fig. 1 depicts the two subassemblies along with the measurements instruments locations. Table I summarized some of the important parameters of XX09 and XX10 [10]. 

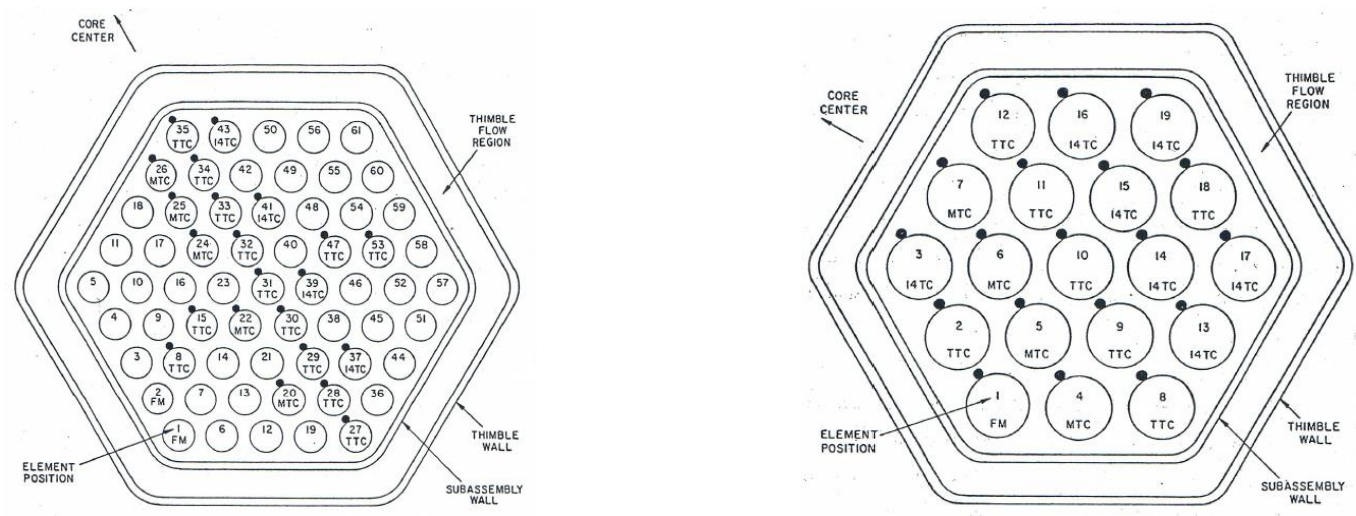

Figure 1. Instrumented subassembly XX09 (left), XX10 (right); FM (flow meter), MTC Midcore cladding TC, TTC Top of core cladding TC, 14 TC Above core TC [10]

Table I. Design parameters of the instrumented subassemblies XX09 and XX10

\begin{tabular}{|c|c|c|}
\hline & XX09 & $\mathrm{XX10}$ \\
\hline Fuel alloy (wt \%) & $\mathrm{U}-5 \mathrm{Fs}$ & Stainless Steel \\
\hline Enrichment (wt\% U235) & 67 & $\mathrm{~N} / \mathrm{A}$ \\
\hline Number of elements & $59 / 61$ & $18 / 19$ \\
\hline Fuel-slug length (m) & 0.3429 & N/A \\
\hline Fuel-slug diameter (mm) & 3.36 & N/A \\
\hline Cladding outer diameter $(\mathrm{mm})$ & 4.41 & 8.81 \\
\hline Pin pitch $(\mathrm{mm})$ & 5.664 & 10.058 \\
\hline Element length $(\mathrm{m})$ & 0.6108 & 0.6108 \\
\hline Cladding material & SS316 & SS316 \\
\hline Spacer-wire diameter $(\mathrm{mm})$ & 1.24 & 1.24 \\
\hline Spacer-wire material & SS316 & SS316 \\
\hline MTC axial location (m) & 0.172 & 0.172 \\
\hline TTC axial location $(\mathrm{m})$ & 0.322 & 0.322 \\
\hline 14TC axial location (m) & 0.48 & 0.48 \\
\hline
\end{tabular}

\section{RESULTS AND ANALYSIS}

Subassemblies XX09 and XX10 were simulated with CTF under SHRT-17 and SHRT-45 conditions. The boundary conditions were based on the data provided from the benchmark specifications [10]. The input file flag 'iproperties' was set to direct the code to call the sodium properties. The code was modified to accept the SFRs characteristics that are different from LWRs (higher operation temperatures at lower pressure) without crashing. The outlet pressure of the subassemblies was set to atmospheric pressure. The flags 'imix' and 'fricfactor' were set to model the flow mixing and the pressure losses for wire-wrapped fuel pins. In this work, the steady state conditions were simulated to evaluate the accuracy of the implementation of sodium coolant and SFR models used and as a proof of the ability of CTF to model SFRs. 


\subsection{SHRT-17}

The boundary conditions for XX09 and XX10 were obtained from the benchmark data. For XX09, the flow rate was $2.377 \mathrm{~kg} / \mathrm{s}$ and the inlet temperature was $356.5^{\circ} \mathrm{C}$. The flow rate in the thimble region was taken to be $0.256 \mathrm{~kg} / \mathrm{s}$ [15]. The total assembly power was provided from the benchmark data and were used to compute the Linear Heat Rates (LHR). The average LHR was $23.44 \mathrm{~kW} / \mathrm{m}$ at the active fuel length locations with a sinusoidal shape and an axial power peaking factor of 1.35 . The flow rate for XX10 was $0.319 \mathrm{~kg} / \mathrm{s}$ and the average LHR was axially uniform and equal to $1.55 \mathrm{~kW} / \mathrm{m}$. The inlet temperature was $353.85^{\circ} \mathrm{C}$. Fig. 2 illustrates the results for XX09 subassemblies at the thermocouples locations. The results were compared to the data in open literature provided by several benchmark participant institutions. The literature data were extracted using WebPlotDigitzer app [18]. That might create some errors but it does not affect the overall comparison conclusions. ENEA (Italian National Agency for New Technologies, Energy and Sustainable Economic Development, Italy) and IGCAR (Indira Gandhi Centre for Atomic Research, India) used CFD methods [15], [16], while TIT/U-Fukui (University of Fukui, Japan) used a version of the subchannel code COBRA-IV [14].

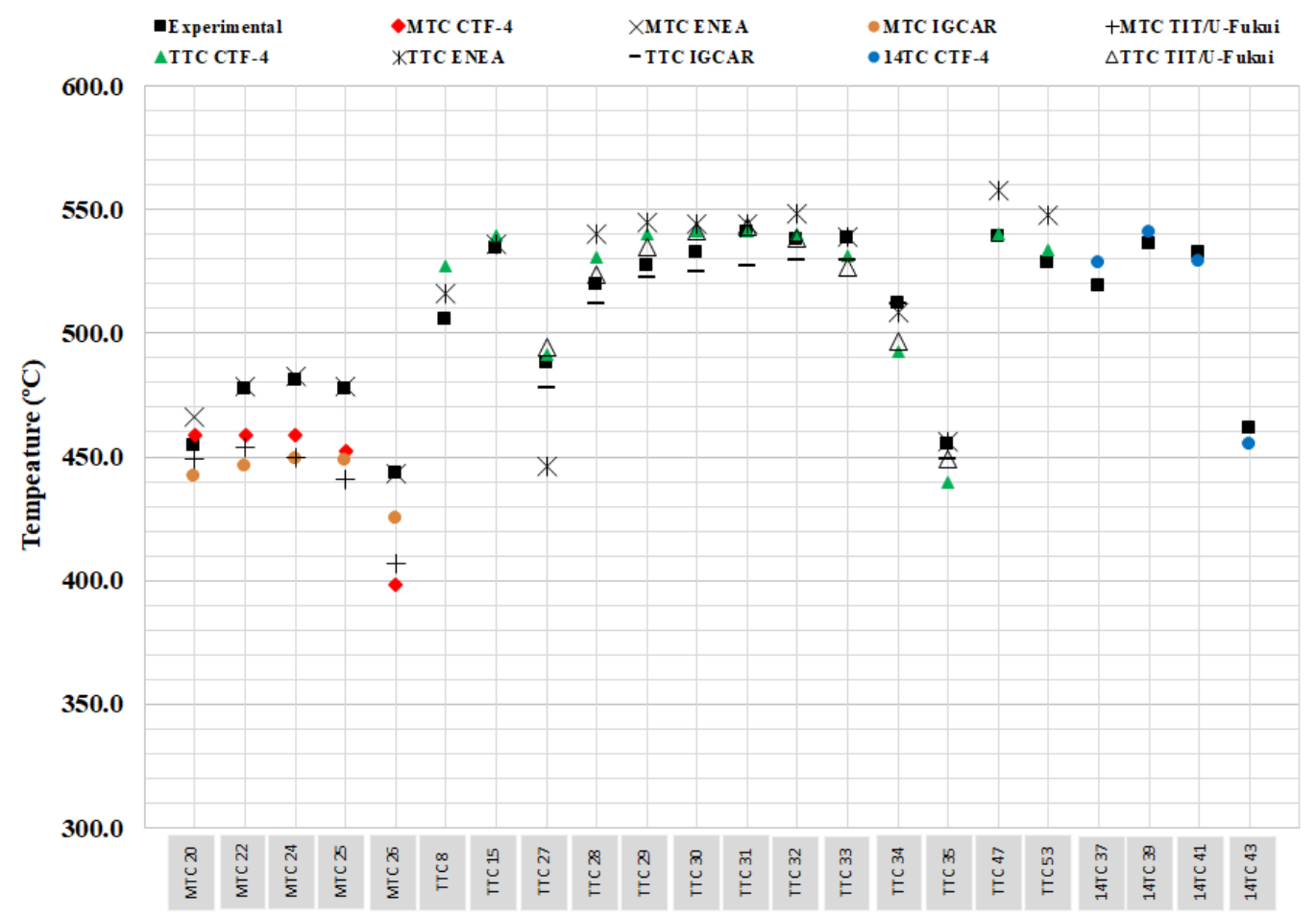

Figure 2. SHRT-17 steady state temperature predictions for XX09 subassembly.

It can be noticed in Fig. 2 that at Mid-core the temperature is underestimated by a maximum of $30^{\circ} \mathrm{C}$ except for rod 26 location where it reaches $45^{\circ} \mathrm{C}$. This location is at the outer most location in the subassembly and is affected by more cooling through the duct wall to the sodium flowing in the thimble region. There is a good agreement between the results and the experiments at the TTC locations. They compare within a maximum difference of $20^{\circ} \mathrm{C}$. The assembly power was modeled as radially uniform and does not take into consideration the power tilt in XX09 due to the variation of the power levels in the assemblies surrounding it. There is an underprediction of the temperature at the TTC-34 and TTC-35. Those locations are close to the thimble wall which might relate this underprediction to overprediction of the heat transfer close to the 
thimble wall. When the experiment was modeled without the thimble region, the predicted temperatures at those locations were closer to the experimental values. The predicted temperatures at the 14TC locations at the fuel plenum region matches well with the measurements with a maximum difference of $10^{\circ} \mathrm{C}$. Fig. 3 depicts the simulation results of XX10 subassembly. There were no open literature steady state results available for comparison. The figure depicts the results for MTC, TTC, and 14TC locations. CTF results shows good matching with the experiment data within $20^{\circ} \mathrm{C}$.

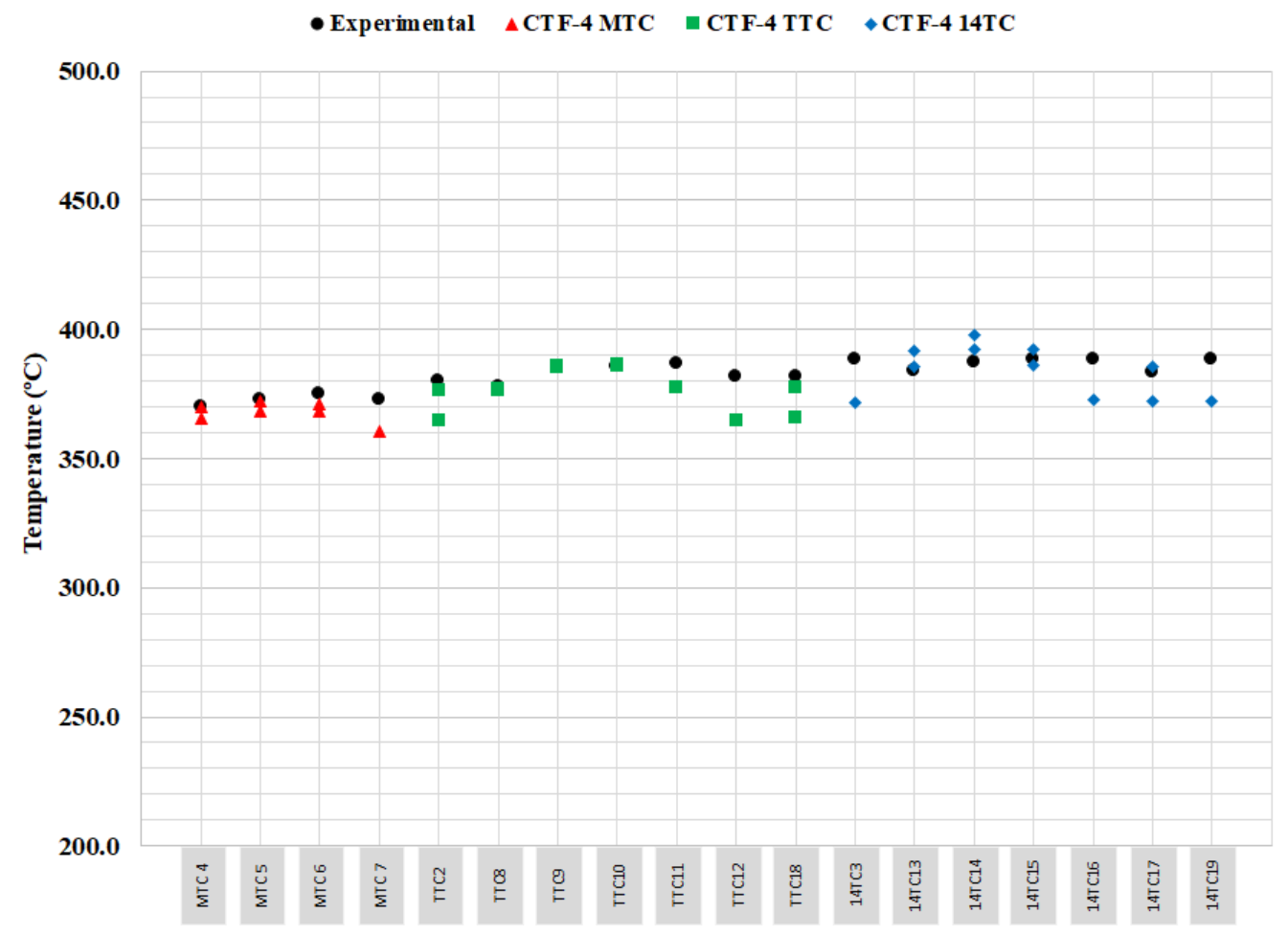

Figure 3. SHRT-17 steady state temperature predictions for XX10 subassembly.

\subsection{SHRT-45}

The boundary conditions for XX09 and XX10 were obtained from the benchmark data. For XX09, the flow rate was $2.2 \mathrm{~kg} / \mathrm{s}$, the inlet temperature was not provided by the benchmark and was set to be same value used for XX09 in SHRT-17. The total assembly power was provided from the benchmark data and were used to compute the LHR. The average LHR was $18.15 \mathrm{~kW} / \mathrm{m}$ with a sinusoidal shape and an axial power peaking factor of 1.25 . The flow rate for XX10 was $0.3 \mathrm{~kg} / \mathrm{s}$ and the LHR was axially uniform $1.57 \mathrm{~kW} / \mathrm{m}$. The inlet temperature was $352.1^{\circ} \mathrm{C}$.

Fig. 4 illustrates the prediction of the TTC temperatures. The maximum difference between CTF and the experimental data was $35^{\circ} \mathrm{C}$. Like SHRT-17, the underprediction of the temperature at TTC-34 and TTC35 might be due to the overprediction of the heat transfer to the thimble region. Since the temperature prediction was generally overestimated at TTC locations, this reflected as well on the prediction at the 14TC locations above the active core region. The maximum difference in the temperature prediction at the 14TC locations was $25^{\circ} \mathrm{C}$. This might be related to errors in the measurement of the coolant mass flow rates in the XX09 subassembly. There were not significant changes in the flow rates and power level in XX10 between SHRT-17 and SHRT-45 and the same analysis applies for SHRT-45 as shown in Fig. 5. 


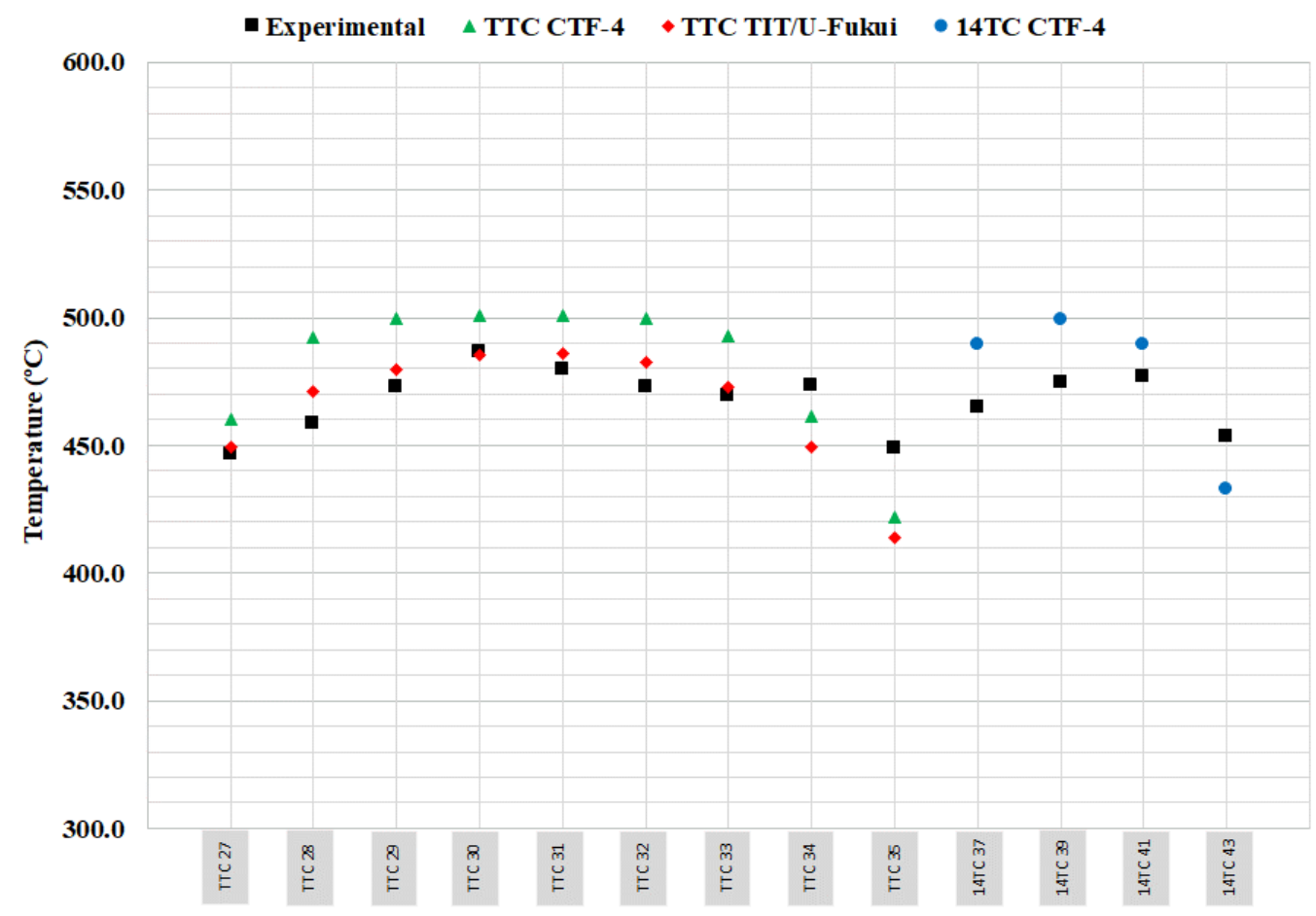

Figure 4. SHRT-45 steady state temperature predictions for XX09 subassembly.

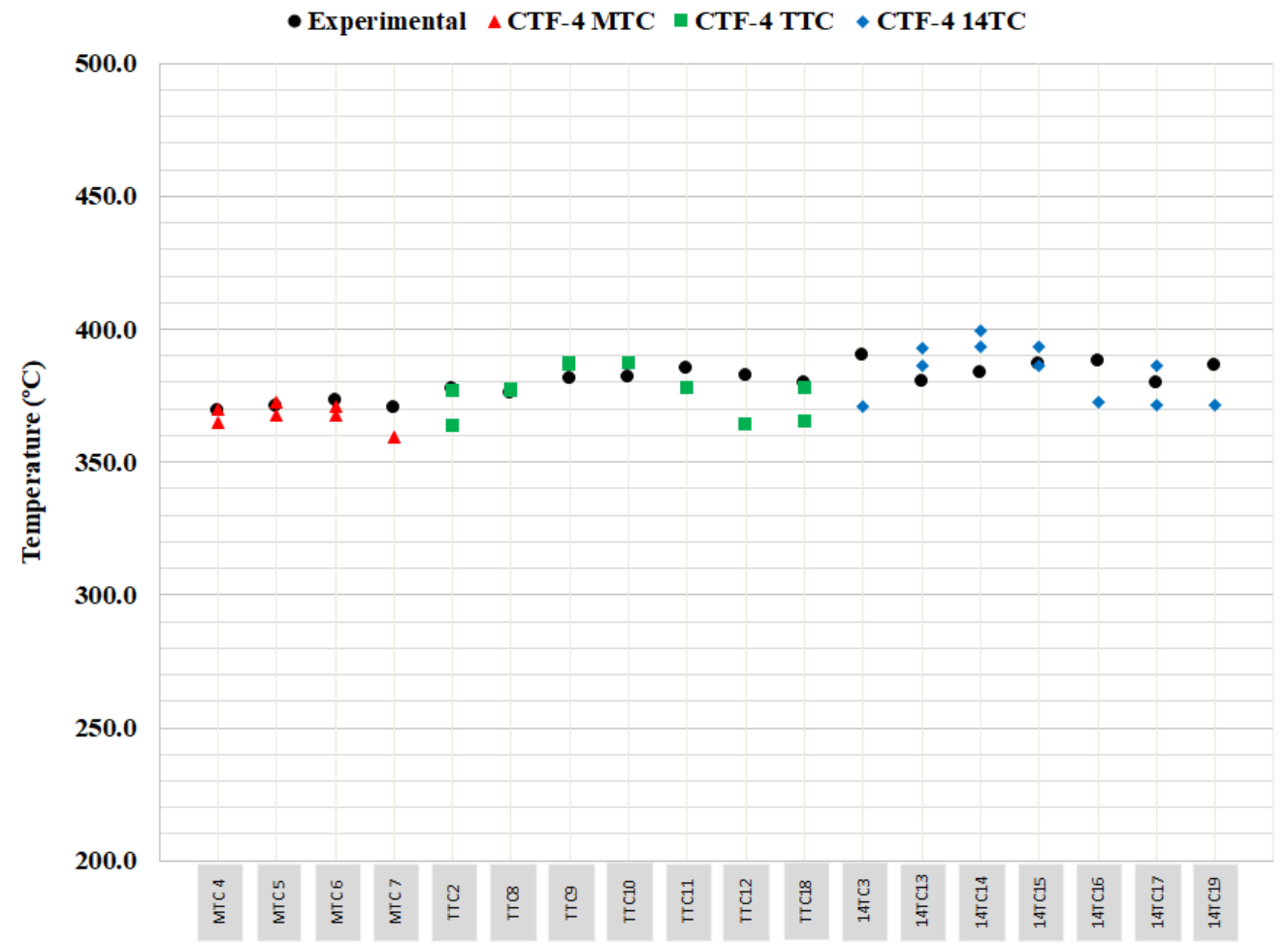

Figure 5. SHRT-45 steady state temperature predictions for XX10 subassembly. 


\section{CONCLUSIONS AND FUTURE WORK}

CTF is well developed and extensively validated code for LWRs. The purpose of this work was to extend the code abilities to model SFRs. For this purpose, the sodium coolant properties were implemented. Correlations by Cheng and Todreas were also added to model the effects of the wire spacers, which are used for mechanical support of the fuel, on the flow mixing and the friction pressure losses. The conduction shape factor was added to the code and the heat conduction between the coolant subchannels was accounted for in the energy equation.

The implementation of the new models was validated using the EBR-II data for SHRT-17 and SHRT-45 tests. Two instrumented subassemblies, XX09 and XX10, provided steady state and transient temperature and flow rate measurements. The steady state conditions of the SHRT experiments were simulated with CTF and a good agreement was observed with an exception of a slight overprediction seen for subassembly XX09 in SHRT-45.

The future work consists of modeling XX09 and XX10 subassemblies during the entire SHRT-17 and SHRT-45 transients.

\section{REFERENCES}

1. Y.S. Tang et al., Thermal Analysis of Liquid-Metal Fast Breeder Reactors, PP. 5, American Nuclear Society, Madison, Pennsylvania, USA (1978).

2. R.L. Sun et al., "Development of a Subchannel Analysis Code for SFR Wire-Wrapped Fuel," Prog. Nucl. Energ., 104, pp. 327-341 (2018).

3. https://gain.inl.gov/SiteAssets/Fast\%20Reactors/SFR-NRCTechnologyandSafetyOverview18Feb15.pdf Last checked 30 Sep. 2019.

4. S.K. Cheng, et al., "Evaluation of Existing Correlations for the Prediction of Pressure Drop in Wire-Wrapped Hexagonal Array Pin Bundles," Nuclear Engineering and Design. 267, pp. 109-131 (2014).

5. S. Pramuditya, M. Takahashi, "Thermal Hydraulics Analysis of Wire-Wrapped SFR Test Subassemblies by Subchannel Analysis Method," Annals of Nuclear Energy, 54, pp. 109-119 (2013).

6. N.E. Todreas, M.S. Kazimi, Nuclear Systems Vol.I Thermal Hydraulic Fundamentals, PP. 451, Taylor and Francis Group, New York, USA (1990).

7. M. Avramova et al., "VERA 3.6 - CTF User's Manual," USA (2017).

8. S.K. Cheng, N.E Todreas, "Hydrodynamics Models and Correlations for Bare and Wire-Wrapped Hexagonal Rod Bundles- Bundle Friction Factors, Subchannel Friction Factors and Mixing Parameters," Nuclear Engineering and Design, 92, pp. 227-251 (1986).

9. L.J. Koch, Experimental Breeder Reactor-II (EBR-II) An Integrated Experimental Fast Reactor Nuclear Power Station, Authorized by Argonne Nation Laboratory, Illinois, USA.

10. T. Sumner, T.Y.C Wei, Benchmark Specifications and Data Requirements for EBR-II Shutdown Heat Removal Tests SHRT-17 and SHRT-45, Nuclear Energy Division, Argonne National Laboratory, Illinois, USA (2012).

11. G. Lerchl, Excerpt of ATHLET Models and Methods, Munich, 2011.

12. J. Hirshhorn, Incorporating Liquid Sodium Analysis Capability into CTF, BSc with Honors, PSU, (2016).

13. H.Y. Jeong et al., "Evaluation of the Conduction Shape Factor with a CFD Code for a Liquid-Metal Heat Transfer in Heated Triangular Rod Bundles," Nuclear Engineering and Design, 237, pp. 648-654 (2007).

14. H. Mochizuki, K. Muranaka, "Benchmark Analyses for EBR-II Shutdown Heat Removal Tests SHRT-17 and SHRT-45 - 2 Subchannel Analysis of Instrumented Fuel Assembly," Nuclear Engineering and Design, 330, pp. 14-27 (2018).

15. A. Del Nevo, et al., Development and Validation of an Approach and Numerical Models for Safety Analysis of FBR, Technical Report APFISS-LP2-088, ENEA, CIRTEN-UNIROMA, Italy (2015).

16. WebPlotDigitizer, "https://apps.automeris.io/wpd/", Last Checked 30 Sep, 2019.

17. IAEA TECDOC SERIES, Benchmark Analysis of EBR-II Shutdown Heat Removal Tests, IAEA-TecDOC-1819, IAEA, Vienna, Austria (2017). 